\title{
No association between water fluoridation and mortality from cancer
}

\author{
McDonagh M, Whiting P, Bradley M, Cooper J, Sutton A, Chestnutt I, et al. A Systematic Review of Public Water \\ Fluoridation. York: Publications Office, NHS Centre for Reviews and Dissemination, University of York. \\ ISBN 190064016 3; 2000
}

Objective Does water fluoridation have negative effects? This objective was broken down into four sections, fluorosis, bone fracture and bone development effects, cancer and other possible adverse effects.

Data sources See page 37.

Study selection Twenty-six studies were included, 18 of level $\mathrm{C}$ and eight of level B (see page 37 ). The mean validity score was 3.8 (range, 2.8-4.8).

Results Incidence of 'all cause' cancer and associated mortality was considered as an outcome in 10 studies and 22 analyses were made. Of these, in only two studies was a significant association found, one a negative association (more cancers) in one of eight subgroups, and in the other a significant positive effect (fewer cancers). Of nine studies comprising 20 analyses of bone cancers, one found a significant negative effect in both men and boys, ie, more cancers. Because of the varying outcome measures, results could not be pooled formally.

\begin{abstract}
Conclusions The findings of cancer studies were mixed, with small variations on either side of no effect. Individual cancers examined were bone cancers and thyroid cancer, where once again no clear pattern of association was seen. Overall, in the research evidence included no association was detected between water fluoridation and mortality from any cancer, or with bone or thyroid cancers specifically.
\end{abstract}

Evidence-Based Dentistry (2002) 3, 47-48. doi:10.1038/sj. ebd.6400108

Address for reprints: Publications Office, NHS Centre for Reviews and Dissemination, University of York, York YO10 5DD, UK. Tel: +44 (0)1904 433648; Fax: +44 (0)1904 433661;

E-mail: crdpub@york.ac.uk; http://www.york.ac.uk/inst/crd/fluorid.htm

\section{Commentary}

Community water fluoridation has been used for cariostatic purposes for more than 50 years, and currently hundreds of millions of people are receiving fluoridated drinking water. A number of populations, particularly those in Africa, Asia and South America, have also lived for many generations in communities where natural fluoride occurs in their water supplies, often at excessive levels. ${ }^{1}$

Cancer remains a major disease and cause of mortality in human beings. The serious effect on health of cancers can have a devastating impact on individual patients and their families, and on public health systems. Water fluoridation and cancer risk has been a controversial and frequently debated issue since the earliest years of community water fluoridation. Because of the significance of the question, there have been several comprehensive reviews of scientific literature on the topic. $^{2-4}$ Using sound scientific approaches, no credible evidence has been detected for the association between fluoridated water ingestion and the risk of cancer.

The present systemic review of water fluoridation and mortality from any cancers included 26 studies that were accepted for analysis using established criteria. Special attention was given to osteosarcoma and bone cancers because fluoride is a bone-seeking element. Thyroid cancers were also analysed separately as fluoride has been suggested to have an effect on the thyroid gland. The results of the well-conducted, rigorous analyses of the studies showed no consistent increase in the mortality from cancers with exposure to fluoridated water. In fact, the review found all three possible associations between water fluoridation and mortality from cancers: decreased risk in 11 analyses, increased risk in nine, and no effect in two studies. The results were similar for osteosarcoma and thyroid cancers.

The contradictory results of the studies are most likely a result of inherent confounding factors in such investigations. Cancer is a multifactorial disease with numerous known mechanisms and sometimes unknown etiology. The risk of developing cancer increases dramatically with age, and cancer mortality differs considerably between men and women and between different ethnic groups. Consequently, cancer incidence in a population can be influenced by small differences in the age, sex and ethnicity.

The findings of the current systemic review are consistent with those of previous reports. ${ }^{2-4}$ The overall available scientific data, including those analysed in the current review, clearly indicate a lack of association between water fluoridation and mortality from cancers. Theoretically, a negative association between the two events may be 
the result of the inability to detect it, or the true non-existence of such an association. Negative (no increase) findings in experimental carcinogenesis research are often considered only to support the conclusion of 'no evidence of increased cancer risk' where it is not possible to discriminate between chemicals that are truly noncarcinogenic and those for which carcinogenicity is simply not detectable with available methods. Logically, however, it is impossible to demonstrate something that does not exist. Therefore, it is imperative that the significance of such a negative or lack of association be recognised and accepted in the analysis of scientific data. Refusal to consider any concept of negativity could lead to the erroneous conclusion that distilled water at $1 \mathrm{ppm}$ concentration would be carcinogenic, as demonstrated by Clayson and Krewski. ${ }^{5}$
In summary, the results of the present systemic review have shown no association between water fluoridation and mortality from cancer which, so far, is the best available and most reliable evidence on this issue. Considering the demonstrated benefits for dental health, water fluoridation should continue and be promoted, unless new evidence indicates otherwise.

1. Li Y. Excessive exposure to natural fluoride and methods to reduce exposure. In Research Needs Workshop: International Collaborative Research on Fluoride. Edited by JJ Clarkson. J Dent Res 2000; 79:902.

2. International Agency for Research on Cancer. Inorganic fluorides. In International Agency for Research on Cancer Monographs on the Evaluation of Carcinogenic Risk of Chemicals to Humans. Some Aromatic Amines, Anthraquinones and Nitroso Compounds, and Inorganic Fluorides Used in Drinking-Water and Dental Preparations. Vol. 27. Lyon: International Agency for Research on Cancer; 1982:235-303.
3. Knox EG. Fluoridation of Water and Cancer: a Review of the Epidemiological Evidence. Report of the Working Party, Department of Health and Social Security. London: Her Majesty's Stationery Office; 1985.

4. National Research Council. Health Effects of Ingested Fluoride. 7. Carcinogenicity of Fluoride. Washington DC: National Academy Press; 1993:109-123.

5. Clayson DB, Krewski D. The concept of negativity in experimental carcinogenesis. International Commission for Protection Against Environmental Mutagens and Carcinogens Working Paper No. 3. Mutation Res 1986; 167:233-240.

Yiming Li Center for Dental Research, School of Dentistry,

Department of Microbiology and Molecular Genetics, School of Medicine, Loma Linda University, Loma Linda, California, USA 